\title{
On the exact solution of the matrix Riccati differential equation *
}

\author{
L. Ntogramatzidis * A. Ferrante** \\ * Department of Mathematics and Statistics, Curtin University, Perth WA \\ 6845, Australia.(E-mail: L.Ntogramatzidis@curtin.edu.au). \\ ** Dipartimento di Ingegneria dell'Informazione, Università di Padova, via \\ Gradenigo, 6/B - I-35131 Padova, Italy. (E-mail:augusto@dei.unipd.it)
}

\begin{abstract}
In this paper we establish explicit closed formulae for the solution of the matrix Riccati differential equation (RDE) with a terminal condition that that involve particular solutions of the associated algebraic Riccati equation. We discuss how these formulae change as assumptions are progressively weakened.
\end{abstract}

\section{INTRODUCTION}

The matrix Riccati differential equation (RDE) is one of the cornerstones of optimal control/filtering theory over finite horizons, see e.g. Bittanti et al. (2008) and the bibliography therein. For example, the solution of the classical finite-horizon linearquadratic (LQ) optimal control problem is traditionally expressed in terms of the solution of a matrix Riccati differential equation with a terminal condition, see e.g. Anderson and Moore (1989).

Starting from the seventies, a significant stream of literature emerged on the problem of finding numerically reliable and efficient algorithms for the integration of the Riccati differential equation, see e.g. Davison and Maki (1973). A popular method is based on the so-called Bernoulli substitution technique, which consists in exploiting the solution of a larger linear differential equation (the so-called Hamiltonian differential equation), whose size is twice the size of the Riccati equation to be solved. Another important class of algorithms is based on the so-called Chandrasekhar decomposition, see Lainiotis (1976) and Kenney and Leipnik (1985), which consists in constructing the solution of the RDE from the solution a pair of coupled matrix linear differential equations.

An area of intense research activity, which originated in the early eighties and flourished in the nineties, was the characterisation of the solutions of the RDE by using non-recursive formulae. The first and most general formula introduced in the literature exploits the solution of the Hamiltonian differential equation via a Bernoulli substitution as described above. However, this formula was soon found to be unsuitable for studying important properties of the solutions of the RDE, such as finite escape times, convergence and mechanisms of attraction. Another drawback of Bernoulli substitutions is the difficulty that arises in determining an expression for the variations of the solution of the Riccati differential equation as an explicit function of variations of some parameters of the problem. Therefore, over the last twenty years, many researchers have focused their attention to finding alternative closed-form representations of the solution of the RDE, see Callier and Wilkin (1996), Callier et al. (1994) and McEneaney (2008). Most of the represen-

\footnotetext{
^ This work was partially supported by the Australian Research Council (Discovery Grant DP0986577).
}

tations proposed so far in the literature exploit the stabilising and anti-stabilising solutions of the associated algebraic Riccati equation. These representations can be used to study the dependence of the solution of the Riccati differential equation on certain parameters of the problem in the cases when the functional dependence of the solution of the associated ARE on such parameters is computable (as it happens, for example, in Colaneri and Ferrante (2006)). The problem associated with these alternative representations is that the extreme solutions of the ARE both exist only if the underlying system (in the case of the Riccati equation arising from the finite-horizon LQ problem) is controllable.

The aim of this paper is to obtain more general representations for the solution of the RDE, by progressively removing the assumptions on the problem data. We begin by considering a formula that is valid under the assumption of sign-controllability, see Shayman (1983) and Scherer (1991). Sign-controllability is the weakest form of controllability for continuous-time systems, in the sense that, in particular, controllable, stabilisable and anti-stabilisable systems are all sign-controllable, but the converse is not necessarily true. Moreover, sign-controllability also constitutes the weakest known assumption for which the associated continuous-time ARE is guaranteed to admit a symmetric solution. Under the assumption of sign-controllability, a formula parameterising all the trajectories originating from the Hamiltonian differential equation, introduced in Ferrante and Ntogramatzidis (2007), is exploited to derive a non-recursive formula for the solution of the RDE. This parameterisation of the trajectories of the Hamiltonian differential equation generalises those proposed in Ferrante et al. (2005) and Ntogramatzidis and Marro (2005) for controllable and stabilisable systems, respectively. In the particular case of stabilisability, it is also shown that using the Kalman controllability decomposition it is possible to reduce the size of the algebraic Riccati equation to be solved. Furthermore, particular cases are also identified where a solution of the ARE exists even when the systems is not sign-controllable. This task is accomplished by introducing a first new sign-controllability form, that gives the largest possible sign-controllable part. 


\section{PROBLEM FORMULATION}

Consider the linear time-invariant (LTI) state differential equation with initial boundary condition

$$
\dot{x}(t)=A x(t)+B u(t), \quad x(0)=x_{0},
$$

where, for all $t \geq 0, x(t) \in \mathbb{R}^{n}$ is the state and $u(t) \in \mathbb{R}^{m}$ is the control input. Matrices $A$ and $B$ in (1) are appropriate dimensional constant matrices. Given the time horizon $[0, T]$, where $T>0$, the objective of the classic (regular) finite-horizon linear-quadratic (LQ) optimal control problem is to find a measurable input function $u(t), t \in[0, T)$, and an absolutely continuous state trajectory $x(t), t \in[0, T]$, that minimise the quadratic performance index

$$
J=\int_{0}^{T}\left[x^{\top}(t) u^{\top}(t)\right]\left[\begin{array}{cc}
Q & S \\
S^{\top} & R
\end{array}\right]\left[\begin{array}{l}
x(t) \\
u(t)
\end{array}\right] d t+x^{\top}(T) P_{T} x(T)
$$

subject to (1). The classic assumptions on matrices $P_{T} \in \mathbb{R}^{n \times n}$, $Q \in \mathbb{R}^{n \times n}, S \in \mathbb{R}^{n \times m}$ and $R \in \mathbb{R}^{m \times m}$ are the following:

(1) the so-called Popov matrix

$$
\Pi \stackrel{\text { def }}{=}\left[\begin{array}{cc}
Q & S \\
S^{\top} & R
\end{array}\right]
$$

and the final state penalty matrix $P_{T}$ are symmetric and positive semidefinite;

(2) matrix $R$ is positive definite.

It is well known that the solution of this problem can be expressed in state-feedback form, i.e., the control function that minimises $J$ subject to (1) can be written as

$$
u(t)=K(t) x(t),
$$

where the time-varying gain

$$
K(t)=-R^{-1}\left(S^{\top}+B^{\top} P(t)\right)
$$

is expressed in terms of the solution $P(t), t \in[0, T]$, of the matrix Riccati differential equation

$$
\begin{array}{r}
\dot{P}(t)+A^{\top} P(t)+P(t) A-(P(t) B+S) R^{-1} \\
\cdot(P(t) B+S)^{\top}+Q=0
\end{array}
$$

with terminal condition

$$
P(T)=P_{T} .
$$

We recall that the Popov triple is defined as the triple $\Sigma \stackrel{\text { def }}{=}$ $(A, B, \Pi)$, see e.g. Ionescu et al. (1999). Using this notation, the $\operatorname{RDE}(2)$ is concisely denoted by $\operatorname{RDE}(\Sigma)$, while the differential problem (2-3) is referred to as the Riccati differential problem and denoted by $\operatorname{RDP}\left(\Sigma, P_{T}\right)$. The solution of $\operatorname{RDE}(\Sigma)$ and $\operatorname{RDP}\left(\Sigma, P_{T}\right)$ are related to the solution $X, \Lambda:(-\infty, T] \longrightarrow \mathbb{R}^{n \times n}$ of the Hamiltonian differential equation $\operatorname{HDE}(\Sigma)$

$$
\left[\begin{array}{c}
\dot{X}(t) \\
\dot{\Lambda}(t)
\end{array}\right]=H\left[\begin{array}{c}
X(t) \\
\Lambda(t)
\end{array}\right]
$$

where

$$
H=\left[\begin{array}{cc}
A-B R^{-1} S^{\top} & -B R^{-1} B^{\top} \\
-Q+S R^{-1} S^{\top} & -A^{\top}+S R^{-1} B^{\top}
\end{array}\right],
$$

and to the solution of the Hamiltonian differential problem $\operatorname{HDP}\left(\Sigma, P_{T}\right)$, i.e., a solution $X(t), \Lambda(t)$ of $\operatorname{HDE}(\Sigma)$ that also satisfies the boundary condition

$$
\left[\begin{array}{l}
X(T) \\
\Lambda(T)
\end{array}\right]=\left[\begin{array}{c}
I_{n} \\
P_{T}
\end{array}\right]
$$

The relation between the solutions of $\operatorname{RDE}(\Sigma)$ and $\operatorname{RDP}\left(\Sigma, P_{T}\right)$ with those of $\operatorname{HDE}(\Sigma)$ and $\operatorname{HDP}\left(\Sigma, P_{T}\right)$ is stated in precise terms as follows.

Theorem 2.1. (Coppel (1974), pp. 274-275)

Let $X, \Lambda:(-\infty, T] \longrightarrow \mathbb{R}^{n \times n}$ be the solutions of the Hamiltonian differential problem $\operatorname{HDP}\left(\Sigma, P_{T}\right)$. Then

(1) $X(t)$ is non-singular for all $t \in(-\infty, T]$;

(2) the solution $P(t)$ of the $\operatorname{RDP}\left(\Sigma, P_{T}\right)$ is

$$
P(t)=\Lambda(t) X^{-1}(t), \quad t \in(-\infty, T] .
$$

If $P(t)$ is a solution of the $\operatorname{RDE}(\Sigma)$ on $(-\infty, T]$ and $X(t)$ : $(-\infty, T] \longrightarrow \mathbb{R}^{n \times n}$ is a solution of

$$
\dot{X}(t)=\left(A-B R^{-1}\left(B^{\top} P(t)+S^{\top}\right)\right) X(t),
$$

then $X(t)$ and $\Lambda(t)=P(t) X(t)$ are a solution of $\operatorname{HDE}(\Sigma)$.

Theorem 2.1 provides a first general way to characterise the solution of both $\operatorname{RDE}(\Sigma)$ and $\operatorname{RDP}\left(\Sigma, P_{T}\right)$. Indeed, linearity of $\operatorname{HDE}(\Sigma)$ yields $\left[\begin{array}{l}X(t) \\ \Lambda(t)\end{array}\right]=e^{H t} C$, where $C \in \mathbb{R}^{2 n \times n}$ is an integration constant that can be found by imposing the boundary condition (5). As such, the solution of $\operatorname{HDP}\left(\Sigma, P_{T}\right)$ is given by

$$
\left[\begin{array}{c}
X(t) \\
\Lambda(t)
\end{array}\right]=e^{H(t-T)}\left[\begin{array}{c}
I \\
P_{T}
\end{array}\right]
$$

If we partition the exponential matrix $e^{H(t-T)}$ as $\left[\begin{array}{lll}\Phi_{1,1}(t) & \Phi_{1,2}(t) \\ \Phi_{2,1}(t) & \Phi_{2,2}(t)\end{array}\right]$, where each $\Phi_{i, j}(t)$ is an $n \times n$ time varying matrix, we can express $X(t)$ and $\Lambda(t)$ as $X(t)=\Phi_{1,1}(t)+\Phi_{1,2}(t) P_{T}$ and $\Lambda(t)=$ $\Phi_{2,1}(t)+\Phi_{2,2}(t) P_{T}$, which enable the solution of $\operatorname{RDP}\left(\Sigma, P_{T}\right)$ to be written as

$$
P(t)=\left(\Phi_{2,1}(t)+\Phi_{2,2}(t) P_{T}\right)\left(\Phi_{1,1}(t)+\Phi_{1,1}(t) P_{T}\right)^{-1} .
$$

This approach for solving the Riccati differential equation is known in the literature as Bernoulli substitution, Kenney and Leipnik (1985); McEneaney (2008), and constitutes the basis of the so-called Davison-Maki numerical method, Davison and Maki (1973). Expression (7) is very general. On the other hand, since there is no explicit way to express the submatrices $\Phi_{i, j}(t)$ as a function of the problem data $\Sigma$, this formula is not suitable for studying properties like convergence, limiting behaviour of the solution and mechanisms of attraction, Callier and Wilkin (1996); Callier et al. (1994). Moreover, (7) does not allow to analyse how the solution varies in terms of perturbation of $\Sigma$ or $P_{T}$, which is a fundamental question in many practical and theoretical problems. For this reason, remarkable efforts have been devoted to finding more explicit representations for the solutions of the Riccati differential equation, see e.g. Callier et al. (1994) and the references therein. In this paper, we present new ways to represent the solution of the Riccati differential problem under mild assumptions that are progressively weakened, and generalise the ones previously introduced in the literature in several directions.

\section{SIGN-CONTROLLABILITY FORMS}

In this paper we are mainly concerned with the assumption of sign-controllability of a system (1), Shayman (1983); Kučera (1991).

Definition 3.1. The pair $(A, B)$ is sign-controllable if the set of uncontrollable eigenvalues of $A$ does not contain pairs of elements in the form $(\lambda,-\bar{\lambda})$. 
Equivalently, we can say that $(A, B)$ is sign-controllable if and only if $\lambda+\bar{\mu}=0$ implies

$$
(\operatorname{rank}[A-\lambda I \mid B]-n)(\operatorname{rank}[A-\mu I \mid B]-n)=0 .
$$

Sign-controllability is the weakest form of controllability: It is weaker than the assumption of reachability, and even than that of stabilisability or anti-stabilisability of the pair $(A, B)$. Indeed, it generically holds even in the extreme case of $B=0$.

When $(A, B)$ is not sign-controllable, two important signcontrollability forms can be defined, that will be used in two different scenarios to determine the exact solution of the RDE even when the sign-controllability assumption does not hold.

\subsection{Sign-controllable first form}

The first sign-controllable form introduced in this paper has the largest possible sign-controllable part. Let $(A, B)$ be in the Kalman controllability form

$$
A=\left[\begin{array}{cc}
A_{u} & 0 \\
A_{u c} & A_{c}
\end{array}\right] \quad \text { and } \quad B=\left[\begin{array}{c}
0 \\
B_{c}
\end{array}\right],
$$

where $\left(A_{c}, B_{c}\right)$ is controllable. Consider a non-singular matrix $T$ such that $T^{-1} A_{u} T$ is in Jordan form, partitioned as $T^{-1} A_{u} T=\operatorname{diag}\left(A_{u}^{\prime}, A_{u}^{\prime \prime}\right)$. The Jordan blocks corresponding to the eigenvalues of $A_{u}$ are divided between $A_{u}^{\prime}$ and $A_{u}^{\prime \prime}$ according to the following rule:

i) the Jordan blocks corresponding to eigenvalues on the imaginary axis are placed in $A_{u}^{\prime}$;

ii) every Jordan block $B_{\lambda}$ corresponding to an eigenvalue $\lambda$ of $A_{u}$ such that $-\lambda$ is not an eigenvalue of $A_{u}$ is in $A_{u}^{\prime \prime}$;

iii) for each pair of eigenvalues $(\lambda,-\lambda)$ of $A_{u}$, we denote the corresponding Jordan blocks by $B_{\lambda}$ and $B_{-\lambda}$. If $\operatorname{dim}\left(B_{\lambda}\right) \leq$ $\operatorname{dim}\left(B_{-\lambda}\right)$, we include $B_{\lambda}$ in $A_{u}^{\prime}$ and $B_{-\lambda}$ in $A_{u}^{\prime \prime}$; Otherwise, we include $B_{-\lambda}$ in $A_{u}^{\prime}$ and $B_{\lambda}$ in $A_{u}^{\prime \prime}$.

In this way, $A_{u}^{\prime \prime}$ has no pairs of eigenvalues in the form $(\lambda,-\lambda)$, nor eigenvalues with zero real part. Now, changing coordinates with $U \stackrel{\text { def }}{=} \operatorname{diag}(T, I)$ gives

$$
\begin{aligned}
U^{-1} A U & =\left[\begin{array}{cc}
T^{-1} A_{u} T & 0 \\
A_{u c} T & A_{c}
\end{array}\right]=\left[\begin{array}{cc|c}
A_{u}^{\prime} & 0 & 0 \\
0 & A_{u}^{\prime \prime} & 0 \\
\hline A_{u c}^{\prime} & A_{u c}^{\prime \prime} & A_{c}
\end{array}\right], \\
U^{-1} B & =\left[\begin{array}{c}
0 \\
0 \\
\hline B_{c}
\end{array}\right],
\end{aligned}
$$

where $\left[\begin{array}{ll}A_{u c}^{\prime} & A_{u c}^{\prime \prime}\end{array}\right] \stackrel{\text { def }}{=} A_{u c} T+A_{c}$. The pair $\left(\left[\begin{array}{cc}A_{u}^{\prime \prime} & 0 \\ A_{u c}^{\prime \prime} & A_{c}\end{array}\right],\left[\begin{array}{c}0 \\ B_{c}\end{array}\right]\right)$ is sign-controllable, since $\left(A_{c}, B_{c}\right)$ is controllable and $A_{u}^{\prime \prime}$ has no mirrored pairs of eigenvalues with respect to the imaginary axis. This definition of sign-controllability form provides a signcontrollable part with the largest possible size.

\subsection{Sign-controllable second form}

Let $(A, B)$ be in the Kalman controllability canonical form (8). Define $\Lambda \stackrel{\text { def }}{=} \sigma\left(A_{u}\right) \cap \sigma\left(-A_{u}\right)$. There exists $T \in \mathbb{R}^{n \times n}$ such that $T^{-1} A_{u} T=\operatorname{diag}\left(A_{u}^{\prime}, A_{u}^{\prime \prime}\right)$ with $\sigma\left(A_{u}^{\prime}\right)=\Lambda$. Changing coordinates with $U \stackrel{\text { def }}{=} \operatorname{diag}(T, I)$ gives (9) where, again, the pair $\left(\left[\begin{array}{cc}A_{u}^{\prime \prime} & 0 \\ A_{u c}^{\prime \prime} & A_{c}\end{array}\right],\left[\begin{array}{c}0 \\ B_{c}\end{array}\right]\right)$ is sign-controllable. Differently from the sign-controllability first form, now for each pair of uncontrollable eigenvalues the corresponding Jordan blocks $B_{\lambda}$ and $B_{-\lambda}$ are both placed in the nonsign-controllable part. Hence, the size of the resulting sign-controllable part is smaller than that obtained using the first sign-controllability decomposition.

Example 3.1. Consider the pair of matrices

$$
A=\left[\begin{array}{cccc}
-2 & 0 & 0 & 0 \\
0 & 2 & 0 & 0 \\
3 & 0 & 1 & 2 \\
1 & -1 & 0 & -1
\end{array}\right], \quad B=\left[\begin{array}{l}
0 \\
0 \\
2 \\
0
\end{array}\right]
$$

By denoting with $e_{i}$ the $i$-th vector of the canonical basis of $\mathbb{R}^{4}$, it is easy to see that the reachable subspace from the origin, i.e., the smallest $A$-invariant subspace containing the image of $B$, is given by the span of $e_{3}$, so by changing coordinates with $T=\left[\begin{array}{llll}e_{1} & e_{2} & e_{4} & e_{3}\end{array}\right]$ we obtain the controllability canonical form

$$
\bar{A}=T^{-1} A T=\left[\begin{array}{ccc|c}
-2 & 0 & 0 & 0 \\
0 & 2 & 0 & 0 \\
1 & -1 & -1 & 0 \\
\hline 0 & 0 & 0 & 1
\end{array}\right], \quad \bar{B}=T^{-1} B=\left[\begin{array}{c}
0 \\
0 \\
0 \\
\hline 2
\end{array}\right] .
$$

The non-controllable eigenvalues are $\{2,-2,-1\}-$ so that the pair is not sign-controllable — and the controllable eigenvalue is 1 . Since the Jordan blocks that correspond to the eigenvalues 2 and -2 have the same dimension, in order to obtain the sign-controllability first form, we can place the blocks that correspond to either the eigenvalues $\{-1,2\}$ or $\{-1,-2\}$ in the sign-controllable part. If we choose the first option, the pair $(\bar{A}, \bar{B})$ is already in the sign-controllability form, as $(\bar{A}, \bar{B})$ can be partitioned as

$$
\bar{A}=\left[\begin{array}{c|ccc}
-2 & 0 & 0 & 0 \\
\hline 0 & 2 & 0 & 0 \\
1 & -1 & -1 & 0 \\
0 & 0 & 0 & 1
\end{array}\right], \quad \bar{B}=\left[\begin{array}{l}
0 \\
0 \\
0 \\
2
\end{array}\right]
$$

In order to construct the second sign-controllability form, notice that both blocks corresponding to the mirrored uncontrollable eigenvalues $\{2,-2\}$ must be part of the nonsigncontrollable part. Hence, the original pair $(A, B)$, partitioned as

$$
A=\left[\begin{array}{cc|cc}
-2 & 0 & 0 & 0 \\
0 & 2 & 0 & 0 \\
\hline 3 & 0 & 1 & 2 \\
1 & -1 & 0 & -1
\end{array}\right], \quad B=\left[\begin{array}{l}
0 \\
0 \\
\frac{2}{0} \\
0
\end{array}\right]
$$

is already written in the second form.

\subsection{The algebraic Riccati equation}

Consider the algebraic Riccati equation $\operatorname{ARE}(\Sigma)$

$$
P A+A^{\top} P-(S+P B) R^{-1}\left(S^{\top}+B^{\top} P\right)+Q=0 .
$$

To any solution $P=P^{\top} \in \mathbb{R}^{n \times n}$ of $\operatorname{ARE}(\Sigma)$ corresponds the closed-loop matrix

$$
A_{P} \stackrel{\text { def }}{=} A-B R^{-1}\left(S^{\top}+B^{\top} P\right) .
$$

The set of eigenvalues $\sigma\left(A_{P}\right)$ of $A_{P}$ is a subset of the spectrum of the Hamiltonian matrix $H$, see Theorem 6 in Molinari (1977). The following esistence properties of solutions to algebraic Riccati equations will be used in the sequel, Molinari (1977) and Willems (1971). 
- If the pair $(A, B)$ is stabilisable and $H$ has no purely imaginary eigenvalues, $\operatorname{ARE}(\Sigma)$ has a unique solution $P^{+}=\left(P^{+}\right)^{\top} \geq 0$ which is stabilising, i.e., all the eigenvalues of $A_{P}$ have strictly negative real part, and $P^{+}$is maximal among the solutions of $\operatorname{ARE}(\Sigma)$

- If $(A, B)$ is anti-stabilisable and $H$ has no eigenvalues on the imaginary axis, $\operatorname{ARE}(\Sigma)$ has a unique solution $P^{-}=\left(P^{-}\right)^{\top} \leq 0$ which is anti-stabilising, i.e., all the eigenvalues of $A_{P}$ have strictly positive real part, and $P^{-}$is minimal among the solutions of the $\operatorname{ARE}(\Sigma)$.

- If $(A, B)$ is controllable and $H$ has no eigenvalues on the imaginary axis, the extreme solutions of $\operatorname{ARE}(\Sigma), P^{+}$and $P^{-}$, both exist. The gap matrix $\Delta \stackrel{\text { def }}{=} P^{+}-P^{-}>0$ satisfies the identity $A_{P^{+}}=-\Delta^{-1} A_{P^{-}}^{\top} \Delta$.

- If $(A, B)$ is sign-controllable, $\operatorname{ARE}(\Sigma)$ admits an unmixed solution $P$, i.e., a solution such that if $\lambda,-\bar{\lambda} \in \sigma\left(A_{P}\right)$ implies $\mathfrak{R} e(\lambda)=0$, Shayman (1983); Scherer (1991). Moreover, given a solution $P=P^{\top}$ of $\operatorname{ARE}(\Sigma)$ and the corresponding closed-loop matrix $A_{P}$ defined in (11), the Lyapunov equation

$$
A_{P} Y+Y A_{P}^{\top}+B R^{-1} B^{\top}=0
$$

has a unique solution $Y=Y^{\top} \in \mathbb{R}^{n \times n}$ if and only if $P$ is strongly unmixed, which means that $A_{P}$ does not contain mirrored pairs, i.e., $\lambda \in \sigma\left(A_{P}\right)$ implies $-\bar{\lambda} \notin \sigma\left(A_{P}\right)$, Ferrante and Ntogramatzidis (2007). Since $A_{P}$ is real, this is equivalent to the fact that $\sigma\left(A_{P}\right)$ does not contain opposite pairs $(\lambda,-\lambda)$. Clearly, if $P$ is unmixed and none of the eigenvalues of $A_{P}$ lay on the imaginary axis, then $P$ is also strongly unmixed.

We now exploit the fact that sign-controllability is the weakest system-theoretic assumption on (1) for which the algebraic Riccati equation is known to admit a symmetric solution.

\section{EXACT SOLUTION OF RDE UNDER SIGN-CONTROLLABILITY ASSUMPTION}

The following theorem establishes an explicit expression for the solution of the Riccati differential equation with terminal condition under the sign-controllability assumption.

Theorem 4.1. Assume that $(A, B)$ is sign-controllable and $H$ has no eigenvalues on the imaginary axis. Let $P$ be a strongly unmixed solution of $\operatorname{ARE}(\Sigma)$, let $A_{P}$ be given by (11) and $Y$ be the solution of (12). The matrix $P(t)=\Lambda(t) X^{-1}(t)$, with

$$
\begin{aligned}
& X(t)=e^{-A_{P}(T-t)}\left(I_{n}-Y \tilde{P}\right)+Y e^{A_{P}^{\top}(T-t)} \tilde{P}, \\
& \Lambda(t)=P e^{-A_{P}(T-t)}\left(I_{n}-Y \tilde{P}\right)+\left(P Y-I_{n}\right) e^{A_{P}^{\top}(T-t)} \tilde{P},
\end{aligned}
$$

where $\tilde{P} \stackrel{\text { def }}{=} P-P_{T}$ is the solution of $\operatorname{RDP}\left(\Sigma, P_{T}\right)$.

Proof: Sign-controllability of $(A, B)$ guarantees that an unmixed solution $P$ of $\operatorname{ARE}(\Sigma)$ exists. In view of the absence of purely imaginary eigenvalues in $H, P$ is also strongly unmixed, since $\sigma\left(A_{P}\right) \subset \sigma(H)$. Let $A_{P}$ be the corresponding closed-loop system matrix and $Y$ be the corresponding solution of (12). By adapting the proof of Theorem 1 in Ferrante and Ntogramatzidis (2007), it is easy to see that the set of trajectories of $\operatorname{HDE}(\Sigma)$ is parameterised in $\Phi, \Psi \in \mathbb{R}^{n \times n}$ as

1 Maximal and minimal are here referred to the standard ordering of symmetric matrices, i.e., $M_{1} \geq M_{2}$ if and only if $M_{1}-M_{2} \geq 0$.

$$
\left[\begin{array}{l}
X(t) \\
\Lambda(t)
\end{array}\right]=\left[\begin{array}{l}
I_{n} \\
P
\end{array}\right] e^{A_{P} t} \Phi+\left[\begin{array}{c}
Y \\
P Y-I_{n}
\end{array}\right] e^{A_{P}^{\top}(T-t)} \Psi .
$$

By imposing the boundary conditions $X(T)=I_{n}$ and $\Lambda(T)=P_{T}$ on (15), we find $\Phi=e^{-A_{P} T}\left(Y P_{T}-Y P+I_{n}\right)$ and $\Psi=P-P_{T}$. Thus, the corresponding $X(t)$ and $\Lambda(t)$ are given by (13) and (14). Hence, in view of Theorem 2.1, $X(t)$ is invertible for all $t \in(-\infty, T]$, and $P(t)=\Lambda(t) X^{-1}(t)$ is the solution of $\operatorname{RDP}\left(\Sigma, P_{T}\right)$.

Example 4.1. Consider system (1) with

$$
A=\left[\begin{array}{c|cc}
3 & 0 & 1 \\
\hline 0 & 2 & 0 \\
0 & 0 & -3
\end{array}\right], \quad B=\left[\begin{array}{c}
1 \\
0 \\
0
\end{array}\right] .
$$

This system has uncontrollable eigenvalues $\{2,-3\}$, and therefore is neither controllable, nor stabilisable, nor anti-stabilisable. However, it is clearly sign-controllable. Consider the weight matrices $Q=\operatorname{diag}\{1,2,0\}, S=\left[\begin{array}{lll}1 & 0 & 0\end{array}\right]^{\top}$ and $R=1$, along with $P_{T}=\operatorname{diag}\{2,1,2\}$ and the time horizon $[0, T]$ where $T=4 \mathrm{sec}$. It is easy to see that the only symmetric solutions of the ARE are given by $P_{1}=\operatorname{diag}(0,-1 / 2,0)$ and by the family of matrices

$$
\mathscr{P}=\left\{\left[\begin{array}{ccc}
4 & p & \frac{4}{5} \\
p & \frac{p^{2}-2}{4} & \frac{p}{5} \\
\frac{4}{5} & \frac{p}{5} & \frac{12}{75}
\end{array}\right] \text { with } \quad p \in \mathbb{R}\right\} .
$$

Let us consider the second solution $P \in \mathscr{P}$ for some unspecified value of $p$. The corresponding closed-loop matrix is

$$
A_{P}=\frac{1}{5}\left[\begin{array}{ccc}
-10 & -5 p & 1 \\
0 & 10 & 0 \\
0 & 0 & -15
\end{array}\right]
$$

The corresponding symmetric set of solutions of the Lyapunov equation (12) is

$$
\mathscr{Y}=\left\{\left[\begin{array}{ccc}
\frac{1-2 p y}{4} & y & 0 \\
y & 0 & 0 \\
0 & 0 & 0
\end{array}\right] \text { with } \quad y \in \mathbb{R}\right\} .
$$

Using Theorem 4.1, choosing any $Y \in \mathscr{Y}$ we compute

$$
\begin{aligned}
& X(t)=\left[\begin{array}{ccc}
\frac{e^{2 t}}{2 e^{8}}+\frac{e^{8}}{2 e^{2 t}} & 0 & \frac{e^{2 t}}{5 e^{8}}-\frac{e^{12}}{5 e^{3 t}} \\
0 & \frac{e^{2 t}}{e^{8}} & 0 \\
0 & 0 & \frac{e^{12}}{e^{3 t}}
\end{array}\right], \\
& \Lambda(t)=\left[\begin{array}{ccc}
\frac{2 e^{8}}{e^{2 t}} & 0 & 0 \\
0 & \frac{3 e^{8}}{2 e^{2 t}}-\frac{e^{2 t}}{2 e^{8}} & 0 \\
\frac{2 e^{8}}{5 e^{2 t}}-\frac{2 e^{3 t}}{5 e^{12}} & 0 & \frac{2 e^{3 t}}{e^{12}}
\end{array}\right] .
\end{aligned}
$$

These matrices do not depend on the parameters $p$ and $y$ and, as we expected, $X(t)$ is invertible for all $t \in[0,4]$. Moreover, $\Lambda(4) X^{-1}(4)=P_{T}$. The solution of the RDE with terminal condition $P(T)=P_{T}$ is given by $\Lambda(t) X^{-1}(t)$ for all $t \in(-\infty, T]$.

Now we show how the sign-controllability first decomposition can be used to solve the RDE. Consider the matrix differential Riccati equation (2). Consider the change of coordinate matrix $T_{S}$ that transforms the pair $(A, B)$ into the sign-controllability first form. Define the new Popov triple $\Sigma_{s}$ characterised by $T_{s}^{-1} A T_{s}, T_{s}^{-1} B, T_{s}^{\top} Q T, T^{\top} S$ and $R$. It is easily seen that, given a solution $P_{s}(t)$ of $\operatorname{RDE}\left(\Sigma_{s}\right)$, the matrix $T_{s}^{-\top} P_{s}(t) T_{s}^{-1}$ is a solution of $\operatorname{RDE}(\Sigma)$, and vice-versa. Moreover, in order to obtain the solution $P(t)$ of $\operatorname{RDP}(\Sigma)$ with boundary condition 
$P(T)=P_{T}$, one can solve $\operatorname{RDP}\left(\Sigma_{s}\right)$ in $P_{S}(t)$, with boundary condition $P_{s}(T)=T_{s}^{\top} P_{T} T_{s}$, and then set $P(t)=T_{s}^{-\top} P_{s}(t) T_{s}^{-1}$.

Keeping this in mind, we show that if $(A, B)$ is not signcontrollable, but the nonsign-controllable part is unobservable from the Popov matrix, a parameterisation of the solutions of $\operatorname{HDE}(\Sigma)$ can still be established. Let $(A, B)$ be in the signcontrollability first form as constructed above, i.e.,

$$
A=\left[\begin{array}{cc}
A_{r} & 0 \\
A_{r s} & A_{s}
\end{array}\right] \quad \text { and } \quad B=\left[\begin{array}{c}
0 \\
B_{s}
\end{array}\right],
$$

where $\left(A_{s}, B_{S}\right)$ is sign-controllable; let $n_{s}$ be the order of $A_{s}$, and let $n_{r} \stackrel{\text { def }}{=} n-n_{s}$ be the dimension of the nonsign-controllable part. Let the nonsign-controllable subsystem be unobservable from the Popov matrix, i.e., $Q$ and $S$ are partitioned as $Q=$ $\operatorname{diag}\left(0_{n_{r} \times n_{r}}, Q_{s}\right)$ and $S=\left[\begin{array}{ll}0_{n_{r} \times m} & S_{s}^{\top}\end{array}\right]^{\top}$ and $A_{r s}=0$. If the Hamiltonian matrix referred to the sign-controllable part

$$
H_{s} \stackrel{\text { def }}{=}\left[\begin{array}{cc}
A_{s}-B_{s} R^{-1} S_{s}^{\top} & -B_{s} R^{-1} B_{s}^{\top} \\
-Q_{s}+S_{s} R^{-1} S_{s}^{\top} & -A_{s}^{\top}+S_{s} R^{-1} B_{s}^{\top}
\end{array}\right]
$$

has no eigenvalues on the imaginary axis, a matrix $P_{S}$ and a matrix $Y_{s}$ exist satisfying the ARE referred to the sole signcontrollable part

$$
P_{s} A_{s}+A_{s}^{\top} P_{s}-\left(S_{s}+P_{s} B_{s}\right) R^{-1}\left(S_{s}^{\top}+B_{s}^{\top} P_{s}\right)+Q_{s}=0
$$

and the corresponding closed-loop Lyapunov equation

$$
A_{P_{s}} Y_{S}+Y_{S} A_{P_{s}}^{\top}+B_{S} R^{-1} B_{s}^{\top}=0
$$

respectively, where $A_{P_{s}} \stackrel{\text { def }}{=} A_{s}-B_{s} R^{-1}\left(S_{s}^{\top}+B_{s}^{\top} P_{s}\right)$. As such, $P=\operatorname{diag}\left(0_{n_{r} \times n_{r}}, P_{s}\right)$ and $Y=\operatorname{diag}\left(0_{n_{r} \times n_{r}}, Y_{s}\right)$ are the solutions of $\operatorname{ARE}(\Sigma)$ and of the closed-loop Lyapunov equation referred to the complete system, respectively, as can be proved by a simple substitution. By using these matrices in (15), we obtain a parameterisation of the trajectories of $\operatorname{HDE}(\Sigma)$, and therefore the result established in Theorem 4.1 can still be applied in this case using $P$ and $Y$ obtained above. The fact that $H_{S}$ has no eigenvalues on the imaginary axis is necessary to ensure that a solution $Y_{s}$ of (17) exists.

\subsection{Stabilisability}

The case where the pair $(A, B)$ is stabilisable is encompassed by the sign-controllability assumption, as in such case one can choose the stabilising solution $P^{+}$as strongly unmixed solution of $\operatorname{ARE}(\Sigma)$, and the result in Theorem 4.1 still applies with $P=P^{+}$and $A_{P}=A_{P^{+}}$. However, when $(A, B)$ is stabilisable, an alternative expression for the solution of the $\operatorname{RDE}(\Sigma)$ and $\operatorname{RDP}\left(\Sigma, P_{T}\right)$ can be found by resorting to two elements of the lattice of all symmetric solutions of the ARE restricted to the sole controllable part. This method has the computational advantage of reducing the order of the involved ARE, which, being the sole nonlinear matrix equation to be solved in this procedure, is the sole critical element in terms of numerical robustness.

Let $\mathscr{R}$ be the reachable subspace from the origin, whose dimension is $n_{c}$; let $n_{u} \stackrel{\text { def }}{=} n-n_{c}$. Consider a change of basis matrix $T_{s}$ such that its first $n_{c}$ columns span $\mathscr{R}$. In the new basis, $A, B$ are partitioned as in (8), while $Q$ and $S$ are partitioned as

$$
Q=\left[\begin{array}{cc}
Q_{u} & Q_{u c} \\
Q_{u c}^{\top} & Q_{c}
\end{array}\right] \quad \text { and } \quad S=\left[\begin{array}{c}
S_{u} \\
S_{c}
\end{array}\right] .
$$

The strongly unmixed solution $P^{+}$of $\operatorname{ARE}(\Sigma)$ can be partitioned correspondingly as

$$
P^{+}=\left[\begin{array}{cc}
P_{u}^{+} & P_{u c}^{+} \\
\left(P_{u c}^{+}\right)^{\top} & P_{c}^{+}
\end{array}\right],
$$

and it is easily seen that $P_{c}^{+}$is a solution of the algebraic Riccati equation of the sole controllable part

$$
P_{c}^{+} A_{c}+A_{c}^{\top} P_{c}^{+}-\left(S_{c}+P_{c}^{+} B_{c}\right) R^{-1}\left(S_{c}+P_{c}^{+} B_{c}\right)^{\top}+Q_{c}=0 .
$$

On the other hand, since the pair $\left(A_{c}, B_{c}\right)$ appearing in the former is controllable, the ARE admits an anti-stabilising solution $P_{c}^{-}$, such that $\sigma\left(A_{P_{c}^{+}}\right)=-\sigma\left(A_{P_{c}^{-}}\right)$. Moreover, the gap matrix $\Delta_{c} \stackrel{\text { def }}{=} P_{c}^{+}-P_{c}^{-}$is positive definite, and therefore invertible.

Define also the two $n \times n$ matrices $A^{\star}$ and $P^{\star}$ as

$$
\begin{aligned}
& A^{\star} \stackrel{\text { def }}{=}\left[\begin{array}{cc}
-A_{u}^{\top}-Q_{u c}^{\top}+S_{u} R^{-1} S_{c}^{\top}-A_{u c}^{\top} P_{c}^{-}+S_{u} R^{-1} B_{c}^{\top} P_{c}^{-} \\
0 & A_{c}-B_{c} R^{-1}\left(B_{c}^{\top} P_{c}^{-}+S_{c}^{\top}\right)
\end{array}\right] \\
& \text { and } P^{\star} \stackrel{\text { def }}{=} \operatorname{diag}\left(I_{n_{u}}, P_{c}^{-}\right) \text {. Finally, let } J \stackrel{\operatorname{def}}{=} \operatorname{diag}\left(0_{n_{u} \times n_{u}}, I_{n_{c}}\right) .
\end{aligned}
$$

Theorem 4.2. Assume that $(A, B)$ is stabilisable and $H$ has no eigenvalues on the imaginary axis. The matrix

$$
P(t)=T_{s}^{-\top} \Lambda(t) X^{-1}(t) T_{s}^{-1},
$$

with

$$
\begin{aligned}
& X(t)=e^{A_{P^{+}} t} \hat{\Phi}+J e^{A^{\star}(t-T)} \hat{\Psi} \\
& \Lambda(t)=P^{+} e^{A_{P^{+}} t} \hat{\Phi}+P^{\star} e^{A^{\star}(t-T)} \hat{\Psi}
\end{aligned}
$$

where

$$
\hat{\Phi} \stackrel{\text { def }}{=} e^{-A_{P^{+}} T}\left(I_{n}+\left[\begin{array}{cc}
0 & 0 \\
0 & \Delta_{c}^{-1}
\end{array}\right]\left(T_{s}^{\top} P_{T} T_{s}-P^{+}\right)\right)
$$

and

$$
\hat{\Psi} \stackrel{\text { def }}{=}\left[\begin{array}{cc}
I_{n_{u}} & -P_{u c}^{+} \Delta_{c}^{-1} \\
0 & -\Delta_{c}^{-1}
\end{array}\right]\left(T_{s}^{\top} P_{T} T_{s}-P^{+}\right)
$$

is the solution of $\operatorname{RDP}\left(\Sigma, P_{T}\right)$.

Proof: The functions $X, \Lambda:(-\infty, T] \longrightarrow \mathbb{R}^{n \times n}$ defined by

$$
\left[\begin{array}{c}
X(t) \\
\Lambda(t)
\end{array}\right]=\left[\begin{array}{c}
I_{n} \\
P^{+}
\end{array}\right] e^{A_{P^{+} t}} \Phi+\left[\begin{array}{c}
J \\
P^{\star}
\end{array}\right] e^{A^{\star}(t-T)} \Psi
$$

represent a parameterisation of all the solutions of the Hamiltonian differential equation (4) in $\Phi$ and $\Psi$. This fact can be proved by direct substitution of $(23)$ into $\operatorname{HDE}(\Sigma)$ written with all submatrices of $H$ partitioned as in (8) and (18). Moreover, all the solutions of the Hamiltonian differential equation (4) can be expressed through (23) for a suitable choice of $\Phi$ and $\Psi$. In fact, the images of $S_{1} \stackrel{\text { def }}{=}\left[\begin{array}{ll}I_{n} & P^{+}\end{array}\right]^{\top}$ and $S_{2} \stackrel{\text { def }}{=}\left[\begin{array}{ll}J & P^{\star}\end{array}\right]^{\top}$ are $H$ invariant subspaces, since $H S_{1}=S_{1} A_{P^{+}}$and $H S_{2}=S_{2} A^{\star}$. The eigenvalues of $H$ restricted to im $S_{1}$ coincide with the eigenvalues of $A_{P^{+}}$, while the eigenvalues of $H$ restricted to $i m S_{2}$ are the eigenvalues of $A^{\star}$. However, from the discussion above it follows that the eigenvalues of $A_{P^{+}}$and those of $A^{\star}$ are opposite, i.e., $\sigma\left(A_{P^{+}}\right)=-\sigma\left(A^{\star}\right)$. As a result, im $S_{1} \cap i m S_{2}=\{0\}$, which implies that (23) represents a set of $2 n^{2}$ linearly independent trajectories of $\operatorname{HDE}(\Sigma)$. To find the solution of $\operatorname{RDP}\left(\Sigma, P_{T}\right)$, we need to find $\Phi$ and $\Psi$ to be used in (23) so that (23) represents the solution of $\operatorname{HDP}\left(\Sigma, P_{T}\right)$. By imposing the boundary conditions in (23) we get $e^{A_{P^{+}} T} \Phi+J \Psi=I_{n}$ and $P^{+} e^{A_{P^{+}} T} \Phi+$ $P^{\star} \Psi=T_{S}^{\top} P_{T} T_{s}$. Matrix $\Phi$ can be computed from the first and 
substituted into the second, to get $\left(P^{\star}-P J\right) \Psi=T_{s}^{\top} P_{T} T_{S}-P^{+}$. Matrix $P^{\star}-P^{+} J$ can be written as

$$
P^{\star}-P^{+} J=\left[\begin{array}{cc}
I_{n_{u}} & -P_{u c}^{+} \\
0 & P_{c}^{-}-P_{c}^{+}
\end{array}\right],
$$

and is easily seen to be invertible, and yields

$$
\Psi=\left[\begin{array}{cc}
I_{n_{u}} & -P_{u c}^{+} \Delta_{c}^{-1} \\
0 & -\Delta_{c}^{-1}
\end{array}\right]\left(T_{s}^{\top} P_{T} T_{s}-P^{+}\right) .
$$

Substition of $\Psi$ into $\Phi=e^{-A_{P^{+}} T}\left(I_{n}-J \Psi\right)$ yields (21).

\section{CONCLUDING REMARKS}

The formulae established in this paper for the solution of the matrix Riccati differential equation, which generalise those proposed so far in the literature, can be used to extend the analysis of finite escape time of the solution of the Riccati differential equation carried out in Callier and Willems (1983), page 1240. Moreover, important properties of the dynamic behaviour of the solution of $\operatorname{RDE}(\Sigma)$, such as convergence and mechanisms of attraction, which have been studied in the literature under the assumptions of controllability and stabilisability, can be generalised using the results presented here.

\section{REFERENCES}

B.D.O. Anderson and J.B. Moore. Optimal Control: Linear Quadratic Methods. Prentice Hall International, London, 1989.

S. Bittanti, A.J. Laub and J.C. Willems. The Riccati Equation. Springer-Verlag, Berlin, 1991.

F.M. Callier and J.L. Willems. Large finite horizon and infinite horizon LQ-optimal control problems. Optimal Control: Applications and Methods, 4:31-45, 1983.

F.M. Callier, J.J. Winkin, and J.L. Willems. Convergence of the time-invariant Riccati differential equation and LQ-problem: mechanisms of attraction. International Journal of Control, 59(4):983-1000, 1994.

F.M. Callier and J.J. Winkin. Asymptotic behavious of the solution of the projection Riccati differential equation. IEEE Transactions on Automatic Control, AC-41(5):646659, 1996.

P. Colaneri and A. Ferrante. Algebraic Riccati equation and $J$-spectral factorization for $\mathscr{H}_{\infty}$ filtering and deconvolution. SIAM J. Contr. and Opt.. Vol. 45, No. 1, pp. 123-145, 2006.

W.A. Coppel. Linear-quadratic optimal control. In Proceedings of the Royal Society of Edinburgh, volume 73A, pages 271289, 1974.

E.J. Davison and M.C. Maki. The numerical solution of the matrix Riccati differential equation. IEEE Transactions on Automatic Control, AC-18(1):71-73, 1973.

A. Ferrante, and L. Ntogramatzidis, "A Unified Approach to the Finite-Horizon Linear Quadratic Optimal Control Problem". European Journal of Control, 13/5: 473-488, 2007.

A. Ferrante, G. Marro, and L. Ntogramatzidis. A parametrization of the solutions of the finite-horizon LQ problem with general cost and boundary conditions. Automatica, 1(8):1359-1366, August 2005.

V. Ionescu, C. Oară, and M. Weiss. Generalized Riccati theory and robust control, a Popov function approach. Wiley, 1999.

C.S. Kenney and R.B. Leipnik. Numerical integration of the Differential Matrix Riccati Equation. IEEE Transactions on Automatic Control, AC-30(10):962-970, 1985.
V. Kučera. Algebraic Riccati equations: Hermitian and definite solutions. in: S. Bittanti et al. (Eds.), The Riccati Equation, Communications and Control Engeneering, Springer, Berlin, 1991.

D.G. Lainiotis. Generalized Chandrasekhar algorithms: Timevarying models. IEEE Transactions on Automatic Control, AC-20, p.728, 1976.

G. Marro, L. Ntogramatzidis, and E. Zattoni. $H_{2}$-optimal rejection with preview in the continuous-time domain, Automatica. 41(5):815-821, 2005. See also the Corrigendum in [Automatica 46 (7) (2010), p. 1241],

W.M. McEneaney. A new fundamental solution for differential Riccati equations arising in control. Automatica, 44:920936, 2008.

B.P. Molinari. The time-invariant linear-quadratic optimal control problem. Automatica, 13:347-357, 1977.

L. Ntogramatzidis, and G. Marro, "A parametrization of the solutions of the Hamiltonian system for stabilizable pairs". International Journal of Control, 78(7): 530-533, May 2005.

C. Scherer. The solution set of the algebraic Riccati Equation and the Algebraic Riccati Inequality. Linear Algebra and Its Applications, 153:99-122, 1991.

M.A. Shayman. Geometry of the algebraic Riccati equation, Part I. SIAM Journal of Control and Optimization, 21:375394, 1983.

J.C. Willems. Least squares stationary optimal control and the algebraic Riccati equation. IEEE Transactions on Automatic Control, AC-16(6):621-634, 1971. 